\title{
Modelo Porcino para Avaliação e Desenvolvimento de Diferentes Dispositivos Coronários Baseados em Cateter: Ferramenta Pré-Clínica Fundamental
}

\author{
Micheli Zanotti Galon1, Celso Kiyochi Takimura², Márcio J. Figueira Chaves³, \\ Julliana Carvalho de Campos ${ }^{4}$, J. Eduardo Krieger ${ }^{5}$, Paulo Sampaio Gutierrez ${ }^{6}$, \\ Francisco Rafael Martins Laurindo ${ }^{7}$, Roberto Kalil Filho ${ }^{8}$, Pedro Alves Lemos Neto ${ }^{9}$
}

\begin{abstract}
RESUMO
Introdução: O modelo experimental porcino tem grande similaridade anatômica e fisiológica com o coração humano, e é de fácil reprodutibilidade, sendo de grande valia para testar novas gerações de stents e balões. Este estudo teve como objetivo analisar o desempenho de um modelo experimental para intervenção coronária percutânea na avaliação de diferentes dispositivos coronários. Métodos: Foram estudados 131 porcos juvenis de granja comercial, sendo 109 fêmeas, pesando $26,4 \pm 3,2 \mathrm{~kg}$, anestesiados, monitorados e ventilados mecanicamente, com acesso vascular obtido por via femoral (dissecção ou punção). Após a cateterização seletiva das artérias coronárias com cateter JR 6 F, procedeu-se à utilização do dispositivo coronário a ser estudado. Os animais foram mantidos sob ventilação mecânica até a recuperação e submetidos a reestudos angiográficos 7, 28, 90 e/ou 180 dias após o procedimento. Após a eutanásia, os corações foram coletados e submetidos a análises macro e microscópicas. Resultados: Foram testados seis stents farmacológicos, dois balões farmacológicos e dois stents não farmacológico. O óbito intraprocedimento não planejado incidiu em 1,5\% dos casos e, no pós-procedimento, em 9,2\%, ocorrendo em um período de 12 horas a 6 dias $(2,3 \pm 1,6$ dias). Além das análises obtidas pela angiografia, foram realizados, durante os procedimentos, ultrassom intracoronário em $20 \%$ e tomografia de coerência óptica em $60 \%$, não sendo observados óbitos relacionados ao emprego dessas ferramentas. Conclusões: $O$ modelo experimental porcino percutâneo mostrou ser reprodu-
\end{abstract}

\section{ABSTRACT}

Porcine Model for the Evaluation and
Development of Catheter-Based Coronary Devices:
an Essential Preclinical Tool

Background: The experimental porcine model is anatomically and physiologically similar to the human heart, it is easily reproducible and very useful to test new stent and balloon generations. This study was aimed at analyzing an experimental model to evaluate different coronary devices for percutaneous coronary intervention. Methods: We evaluated 131 juvenile commercial farm pigs, 109 were female, weighing $26.4 \pm 3.2 \mathrm{~kg}$. They were anesthetized and had mechanical ventilation and monitoring. Vascular access was obtained via the femoral artery by dissection or puncture. The coronary device was used after a selective catheterization of the coronary arteries with a JR $6 \mathrm{~F}$ catheter. Animals were maintained on mechanical ventilation until recovery and were submitted to angiographic evaluation 7, 28, 90 and/or 180 days after the procedure. After euthanasia, the hearts were collected and submitted to macro and microscopic analysis. Results: Six drug-eluting stents, two drug-eluting balloons and two bare-metal stents were tested. Unplanned deaths were observed in $1.5 \%$ of the cases during the procedures and in $9.2 \%$ of the cases after the procedure, occurring within 12 hours to 6 days ( $2.3 \pm 1.6$ days). In addition to angiographic evaluations, intravascular ultrasound and optical coherence tomography were performed during the procedures in $20 \%$ and $60 \%$ of the cases, respectively. There was no deaths related to the use of the devices. Conclusions:

\footnotetext{
1 Mestre. Cardiologista intervencionista do Instituto do Coração do Hospital das Clínicas da Faculdade de Medicina da Universidade de São Paulo. São Paulo, SP, Brasil.

2 Doutor. Cardiologista intervencionista do Instituto do Coração do Hospital das Clínicas da Faculdade de Medicina da Universidade de São Paulo. São Paulo, SP, Brasil.

${ }^{3}$ Biólogo do Instituto do Coração do Hospital das Clínicas da Faculdade de Medicina da Universidade de São Paulo. São Paulo, SP, Brasil. ${ }^{4}$ Biomédica do Instituto do Coração do Hospital das Clínicas da Faculdade de Medicina da Universidade de São Paulo. São Paulo, SP, Brasil. ${ }^{5}$ Professor titular. Diretor do Laboratório de Genética e Cardiologia Molecular do Instituto do Coração do Hospital das Clínicas da Faculdade de Medicina da Universidade de São Paulo. São Paulo, SP, Brasil. ${ }^{6}$ Livre-docente. Médico assistente do Instituto do Coração do Hospital das Clínicas da Faculdade de Medicina da Universidade de São Paulo. São Paulo, SP, Brasil.
}

\begin{abstract}
${ }^{7}$ Livre-docente. Médico assistente do Instituto do Coração do Hospital das Clínicas da Faculdade de Medicina da Universidade de São Paulo. São Paulo, SP, Brasil.

${ }^{8}$ Professor titular. Diretor da Divisão de Cardiologia do Instituto do Coração do Hospital das Clínicas da Faculdade de Medicina da Universidade de São Paulo. São Paulo, SP, Brasil.

${ }^{9}$ Livre-docente. Diretor do Serviço de Hemodinâmica e Cardiologia Intervencionista do Instituto do Coração do Hospital das Clínicas da Faculdade de Medicina da Universidade de São Paulo. São Paulo, SP, Brasil.
\end{abstract}

Correspondência: Pedro Alves Lemos Neto. Avenida Dr. Enéas Carvalho de Aguiar, 44 - Cerqueira César - São Paulo, SP, Brasil - CEP 05403-900 E-mail: pedro.lemos@incor.usp.br

Recebido em: 28/8/13 - Aceito em: 5/11/13 
tível, com desempenho homogêneo entre os vários dispositivos e de baixa mortalidade, sendo ferramenta indispensável na investigação de novos dispositivos coronários.

DESCRITORES: Modelos animais. Intervenção coronária percutânea. Stents farmacológicos.

0 modelo experimental porcino é de grande valia para testar novas gerações de stents e balões, sendo este o modelo mais usado na avaliação dos dispositivos coronários. As principais vantagens desse modelo animal são suas semelhanças anatômicas e fisiológicas com os corações humanos. ${ }^{1}$ A distribuição das artérias coronárias, o suprimento miocárdico por colaterais, o sistema de coagulação e a atividade plaquetária apresentam grande similaridade com os humanos. ${ }^{2-4}$

Apesar da cicatrização em resposta à lesão vascular envolver processos e fases semelhantes, o curso de tempo dos eventos, no suíno, é diferente do humano. Com base nos períodos do desenvolvimento da reestenose após o implante de stents não farmacológicos, uma relação temporal de 1 para 6 (animal para humano) é geralmente observada. ${ }^{5-9}$

Apesar da produção de animais não ser cara, os custos de manuseio e de habitação são elevados. No Brasil, os investimentos dos setores público e privado em pesquisa translacional se justificam ao passo que o objetivo final é a aplicação de tecnologias melhoradas, muitas delas de desenvolvimento nacional, que devem se traduzir em benefícios para os pacientes portadores de doença cardiovascular.

Para participarmos das atividades de pesquisa, desenvolvimento e inovação no campo da intervenção percutânea coronária, alvo de intenso interesse em diversos países do mundo, faz-se necessário termos modelos animais bem estabelecidos e reprodutíveis - visto que esta tem sido uma fase obrigatória na avaliação da eficácia de qualquer novo dispositivo coronário, exigidos pelas agências reguladoras americana (Food and Drug Administration - FDA) e europeia (European Medicines Agency - EMEA). ${ }^{10,11}$

Este estudo teve como objetivo analisar o desempenho de um modelo experimental para intervenção coronária percutânea na avaliação de diferentes dispositivos coronários.

\section{MÉTODOS}

Todos os experimentos foram realizados de acordo com o protocolo aprovado pelo Comitê de Ética em Pesquisa institucional. Foram utilizados, neste estudo, porcos de linhagem doméstica (Sus scrofa domestica, linhagem MS60 EMBRAPA). Todos os animais foram submetidos a exames clínicos antes de iniciar o protocolo
The experimental percutaneous porcine model proved to be reproducible with similar outcomes and low mortality for the different devices tested and is an essential tool for the evaluation of new coronary devices.

DESCRIPTORS: Models, animal. Percutaneous coronary intervention. Drug-eluting stents.

e só aqueles sem sinais de doenças foram utilizados. Os animais foram mantidos em uma granja comercial local, com livre acesso a água e a alimento durante os protocolos. Eles foram aclimatados na Divisão Experimental do Instituto do Coração do Hospital das Clínicas da Universidade de São Paulo (InCor-FMUSP) pelo menos 24 horas antes do cateterismo.

\section{Procedimento anestésico}

Os animais foram sedados antes do procedimento com uma mistura de cloridrato de cetamina $(8 \mathrm{mg} / \mathrm{kg})$ e cloridrato de midazolam $(0,5 \mathrm{mg} / \mathrm{kg})$. Após 10 a 20 minutos, foi obtido um acesso venoso (veia superficial da orelha). A anestesia foi induzida com tiopental de sódio $(12,5 \mathrm{mg} / \mathrm{kg})$ e, em seguida, foi realizada entubação orotraqueal com laringoscópio de lâmina reta longa (tubo de 7 ou 7,5 mm). A anestesia foi mantida com isoflurano $1,5 \%$ a $2,5 \%$ e em oxigênio a $100 \%$ no equipamento anestésico. Antes do procedimento, os suínos receberam injeção intramuscular de penicilina benzatina 1,2 milhão de unidades, para evitar infecções.

Medicações antiagregantes plaquetárias foram iniciadas 24 horas antes do procedimento, sendo elas o ácido acetilsalicílico, nas doses de 300 a 600 mg, e o clopidogrel, de 75 a 150 mg. A dose de manutenção foi de $100 \mathrm{mg}$ e $75 \mathrm{mg}$ para o ácido acetilsalicílico e para o clopidogrel, respectivamente.

\section{Cateterismo cardíaco e monitorizações invasiva e não invasiva}

Os porcos sob anestesia geral foram colocados em posição supina. A saturação de oxigênio foi monitorada pela cauda do animal, e a frequência e o ritmo cardíacos por monitor cardíaco. Após assepsia inguinal, uma bainha vascular $6 \mathrm{~F}$ foi introduzida por visão direta na artéria femoral comum por punção ou dissecção, seguida pela administração de 10 mil unidades de heparina e sendo mantida a monitorização invasiva da pressão arterial.

As artérias coronárias direita e esquerda foram seletivamente canuladas utilizando cateter terapêutico Judkins 6 F (Philips, Eindhoven, Holanda) monitorado por fluoroscopia proporcionada pelo equipamento de angiografia digital Philips BV Pulsera (Philips, Eindhoven, Holanda). Foi administrada injeção intracoronária de nitroglicerina na dose de $200 \mu \mathrm{g}$ e posterior injeção de contraste iodado para a aquisição da angiografia inicial. A projeção angiográfica de eleição, tanto para 
a artéria coronária esquerda como para a direita, foi a oblíqua anterior esquerda $60^{\circ}$.

Em caso de arritmia ventricular aguda, doses em bólus de cloridrato de lidocaína $(2,5$ a $12 \mathrm{mg} / \mathrm{kg})$ foram administradas. Quando a fibrilação ventricular era detectada, cardioversão elétrica foi realizada pela aplicação de 200 a 300 J, com as pás contra a parede anterior do tórax.

\section{Dispositivos testados, reestudo e eutanásia}

Os dispositivos testados foram balões farmacológicos, stents farmacológicos e não farmacológicos. O reestudo angiográfico foi realizado aos 7, 28, 90 e 180 dias, dependendo do protocolo vigente.

Os animais, sob anestesia profunda, foram submetidos à eutanásia, com uma dose letal de cloreto de potássio. Foram realizadas a toracotomia paramediana esquerda e a abertura do pericárdio, sendo as veias cavas, o tronco da artéria pulmonar e a aorta cuidadosamente pinçados e seccionados e, em seguida, foi removido o coração. A seguir, os corações foram lavados com água potável e uma solução de formol a $10 \%$ foi infundida, sob pressão de $100 \mathrm{mmHg}$, na raiz da aorta, para perfusão coronária por 30 minutos. Os segmentos arteriais contendo o stent foram dissecados, removidos e colocados em solução de formol a 10\%, sendo encaminhados para processamento histológico.

\section{Emprego de métodos de imagem intravascular adjuntos}

Antes da realização dos métodos de imagem intravascular, foi administrado nitrato intracoronário (mononitrato de isossorbida 10 a $20 \mathrm{mg}$ ou nitroglicerina 150 a $400 \mu \mathrm{g}$ ), com o intuito de promover a vasodilatação epicárdica e evitar espasmo arterial.

Para a realização do ultrassom intracoronário (USIC), foi utilizado o cateter Atlantis $40 \mathrm{Mhz}$ conectado ao console iLab (ambos da Boston Scientific Inc., Estados Unidos). A aquisição foi realizada durante recuo automático a $0,5 \mathrm{~mm} / \mathrm{s}$.

Na tomografia de coerência óptica (TCO) foi utilizado o sistema OCT M2 (LightLab Imaging, Westford, Estados Unidos), com recuo automatizado do cateter de imagem ImageWire ${ }^{\text {TM }}$ (LightLab Imaging) à velocidade de 1 a $2 \mathrm{~mm} / \mathrm{s}$, após prévia oclusão proximal da artéria coronária com balão Helios ${ }^{\circledR}$ (LightLab Imaging) e infusão de soro fisiológico.

\section{Microscopia eletrônica de varredura}

O segmento da artéria coronária contendo o stent foi dissecado, removido e submetido a uma secção longitudinal, sendo uma das metades enviada para estudo anatomopatológico e a outra para processamento específico e posterior análise em microscópio eletrônico de varredura.

O hemissegmento contendo o stent foi imerso em solução de Karnovsky modificada por 12 horas a $4^{\circ} \mathrm{C}$, lavado em solução tampão de cacodilato de sódio, pósfixado em solução tamponada de tetróxido de ósmio a $1 \%$ e desidratado em séries crescentes de álcoois, até o álcool absoluto. A secagem das amostras foi feita ao ponto crítico no aparelho Balzers CPD 030.

As amostras foram metalizadas com íons de ouro em aparelho Balzers SCD 040 e analisadas no microscópio eletrônico de varredura modelo JSM $7401 \mathrm{~S}$ (JEOL, Japão) com a tomada de imagens com aumento de 25 vezes. As imagens obtidas foram arquivadas e submetidas à análise por meio dos softwares Adobe ${ }^{\circledR}$ Photoshop $^{\circledR}$ versão 7.0 (Adobe Systems Inc.) e ImageJ versão $1.42 \mathrm{q}$ para Windows (NIH, Bethesda, Estados Unidos), sendo mensuradas as áreas de hastes expostas dos stents $\left(\mathrm{mm}^{2}\right)$ e calculados os porcentuais de endotelização para cada tipo de stent (Figura 1).
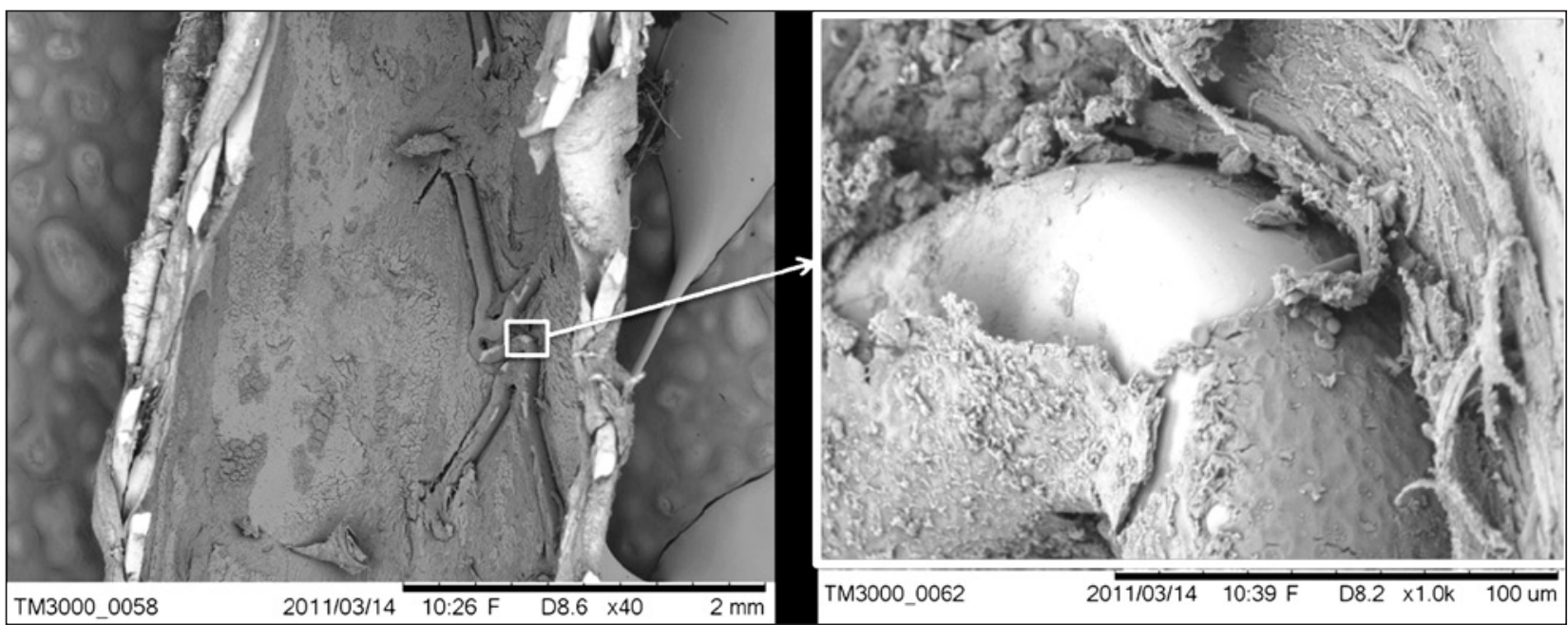

Figura 1. Microscopia eletrônica de varredura da artéria coronária suína 7 dias após o implante de stent farmacológico, na qual observam-se hastes desnudas. No detalhe, imagem da haste com delaminação da cobertura polimérica, magnificada à direita. 


\section{Análise histopatológica}

Os cortes foram corados com hematoxilina-eosina e pela coloração de Verhoeff para fibras elásticas e, posteriormente, analisados qualitativa e quantitativamente por patologistas e técnico especializado. Na análise quantitativa, foram utilizados os escores de inflamação modificada de Kornovski et al., ${ }^{12}$ o escore de fibrina ${ }^{13}$ e os escores de injúria de Schwartz. ${ }^{10,14}$

A quantificação da área do lúmen, da área do stent, da área da lâmina elástica interna, da área da lâmina elástica externa e da espessura neointimal sobre as hastes e inter-hastes foi realizada por meio do software de mensuração Leica QWin (Leica Microsystems, Wetzlan, Alemanha).

\section{RESULTADOS}

Foram estudados 131 porcos juvenis de granja comercial de 2006 a 2012, sendo 109 fêmeas, pesando $26,4 \pm 3,2 \mathrm{~kg}$. Os animais foram mantidos sob ventilação mecânica até a recuperação e submetidos a reestudos após 7, 28, 90 e 180 dias.

Foram testados seis stents farmacológicos, dois baIões farmacológicos e dois stents não farmacológicos (Tabela 1). Óbito não planejado intraprocedimento foi de $1,5 \%$, e pós-procedimento foi de $9,2 \%$, ocorrendo entre 12 horas até 6 dias $(2,3 \pm 1,6$ dias). Além das análises obtidas pela angiografia, foram realizados, durante os procedimentos, USIC em 20\% e TCO em $60 \%$, não sendo observados óbitos durante o emprego dessas ferramentas (Figura 2).

\section{DISCUSSÃO}

O presente estudo reflete os resultados do um centro pioneiro de validação pré-clínica no país, especificamente organizado e equipado para a avaliação científica de dispositivos e procedimentos de intervenção coronária percutânea. O conhecimento adquirido com o modelo porcino nos permitiu, ao longo dos anos, dentre outros dispositivos, participar do desenvolvimento do primeiro stent de concepção brasileira, empregando inovações tecnológicas para sua criação. ${ }^{15-19}$ Tais resultados foram obtidos graças à implementação de inúmeros e complexos processos, que se iniciaram com a concepção de um novo dispositivo endovascular e prosseguiram com as etapas de confecção de protótipos, testes de validação pré-clínicos (mecânicos e biológicos in vitro e in vivo) e, por fim, testes de segurança e eficácia em humanos. ${ }^{20}$

Fez parte deste processo a análise histopatológica, que permanece o padrão vigente para a avaliação dos dispositivos a serem estudados. Tal técnica de processamento histológico de vasos com implante de stent configura como um dos pontos mais limitantes no processo, devido ao risco de danificação dos tecidos adjacentes às hastes do stent. A inclusão em resina

TABELA 1

Descrição dos dispositivos coronários estudados empregando o modelo porcino

\begin{tabular}{|c|c|c|}
\hline Dispositivos estudados & Data & $\mathbf{n}$ \\
\hline Stent liberador de óxido nítrico & Abril de 2006 & 9 \\
\hline Stent nacional de cromo-cobalto & Agosto de 2006 & 10 \\
\hline Stent supralimus vs. sirolimus & Março de 2007 & 3 \\
\hline Stent nacional de cromo-cobalto pré-montado & Setembro de 2007 & 3 \\
\hline Stents metálicos de diferentes modelos & Julho de 2008 & 5 \\
\hline Stent nacional com sirolimus & Outubro de 2008 & 6 \\
\hline Stent nacional com fármaco externo vs. interno e externo & Maio de 2009 & 14 \\
\hline Balões eluidores de sirolimus vs. paclitaxel & Dezembro de 2009 & 7 \\
\hline Stent liberador de óxido nítrico & Março de 2010 & 4 \\
\hline Balão eluidor de sirolimus & Junho de 2010 & 16 \\
\hline Stent eluidor de everolimus vs. eluidor de sirolimus & Agosto de 2010 & 14 \\
\hline Stent nacional de cromo-cobalto vs. nacional com sirolimus 180 dias & Setembro de 2010 & 4 \\
\hline Comparação de stents com coágulo vs. trombo & Novembro de 2010 & 4 \\
\hline Stent nacional de cromo-cobalto vs. sirolimus em overlapping & Dezembro de 2010 & 3 \\
\hline Stent nacional de cromo-cobalto vs. nacional com sirolimus 90 dias & Dezembro de 2010 & 7 \\
\hline Stent nanomagnético & Junho de 2011 & 4 \\
\hline Stent eluidor de sirolimus & Setembro de 2011 & 10 \\
\hline Stent nacional com novo design & Setembro de 2012 & 8 \\
\hline
\end{tabular}



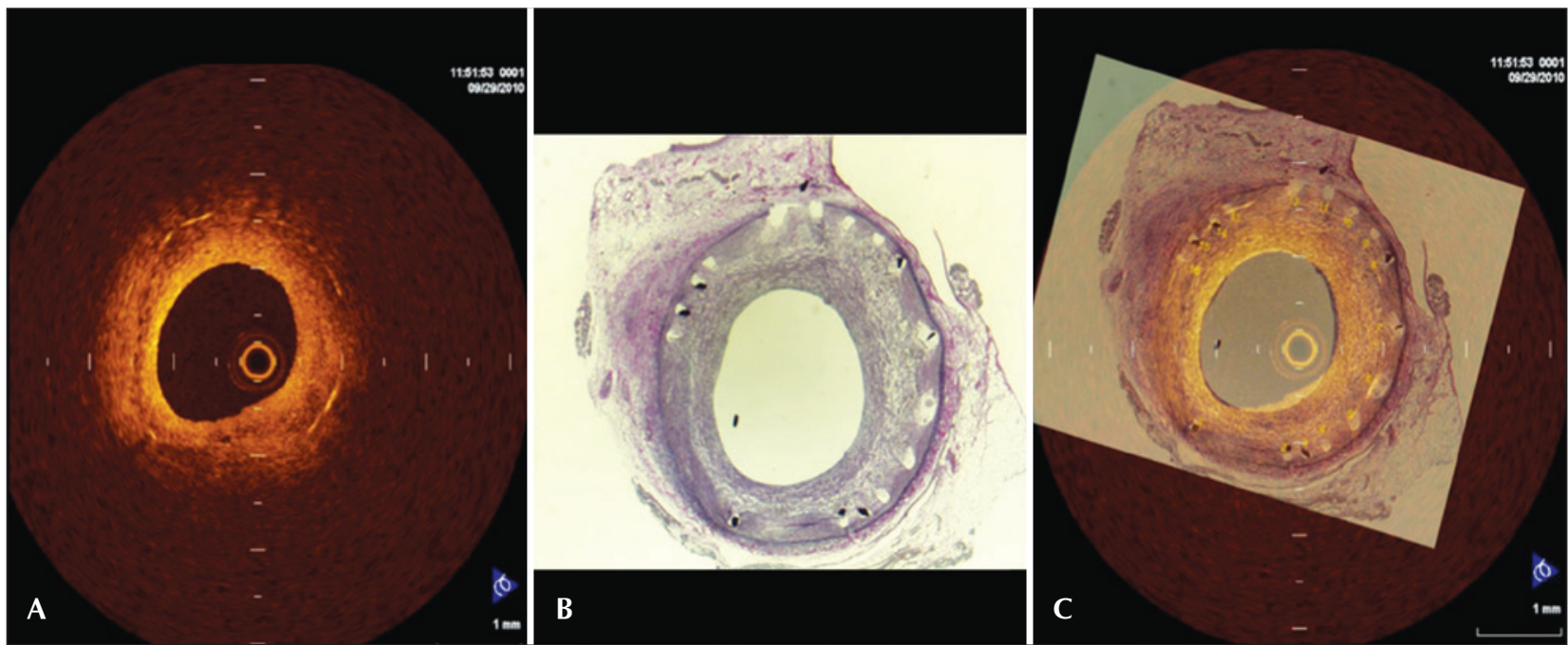

Figura 2. Cortes transversais de artéria coronária suína com stent farmacológico. (A) Tomografia de coerência óptica; (B) histologia; (C) imagens sobrepostas, com a finalidade de escolher seção transversa da tomografia de coerência óptica análoga à imagem histológica.

de metacrilato e o corte micrométrico de espécime contendo metal constituem as principais dificuldades que restringem o processamento histológico de stents a poucos centros no mundo.

Atualmente, recomenda-se que esses modelos, anteriormente utilizados como testes de bancada para a avaliação da eficácia de novos dispositivos, devam concentrar-se especificamente nos aspectos de segurança. ${ }^{21}$ Para tal, o desempenho do stent deve ser avaliado de forma sequencial. Dessa forma, é fundamental dispor de métodos de imagem intravascular que permitam a avaliação evolutiva em diferentes momentos do processo de cicatrização vascular.

O USIC, apesar de fornecer uma estimativa volumétrica de todo tecido neointimal, possui correlação pouco precisa com os resultados histológicos. ${ }^{22,23}$ Logo, esta não é a melhor técnica para avaliar a presença de fibrina ou trombo, nem possui resolução espacial suficiente para verificar se as hastes do stent estão cobertas ou desnudas.

A TCO, por outro lado, mostrou alta correlação com a histologia ${ }^{24,25}$ devido à sua alta resolução axial, permitindo categorizar adequadamente as hastes do stent como cobertas ou desnudas. ${ }^{25,26}$ Imagens heterogêneas, com sinal de baixa intensidade luminosa, visibilizadas na TCO, correlacionaram-se com áreas de baixa celularidade, com presença de fibrina e proteoglicano. ${ }^{19,27,28}$

Atualmente preconiza-se tal avaliação aos 28 dias, para observar a ocorrência de hiperplasia neointimal, e, pelo menos mais uma vez, para analisar os efeitos a longo prazo. A segunda avaliação (depois de 3 a 6 meses) depende da época em que a cicatrização do segmento tratado e a liberação do fármaco tenham sido concluídas. É importante observar que, para os stents farmacológicos, o FDA, que recomendava um intervalo de 6 meses após a implante do stent, para a obtenção dos dados pré-clínicos, mais recentemente estendeu tal recomendação até 1 ano após o implante. ${ }^{10,11}$

\section{CONCLUSÕES}

O modelo experimental porcino percutâneo mostrou ser reprodutível, com comportamento homogêneo entre os vários dispositivos testados e de baixa mortalidade, sendo ferramenta imprescindível na investigação de novos dispositivos coronários.

\section{SUPORTE FINANCEIRO}

Este estudo faz parte do Programa de Desenvolvimento Nacional de Endopróteses Vasculares (stents) (PDNS), iniciado em 2004-2005. Conta com o suporte da Secretaria de Ciência, Tecnologia e Insumos Estratégicos (SCTIE)/Departamento de Ciência e Tecnologia (DECIT) do Ministério da Saúde (MS), do Conselho Nacional de Desenvolvimento Científico e Tecnológico (CNPq) e da Financiadora de Estudos e Projetos (FINEP) do Ministério da Ciência e Tecnologia (MCT). O desenvolvimento do processo de corte a laser de stents contou com o apoio da Fundação de Amparo à Pesquisa do Estado de São Paulo (FAPESP, forma de Apoio: Inovação Tecnológica - Pesquisa Inovativa na Pequena e Microempresa - PIPE).

\section{CONFLITO DE INTERESSES}

Celso Kiyochi Takimura, Francisco Rafael Martins Laurindo e Pedro Alves Lemos Neto são consultores científicos da Scitech Prod Médicos Ltda. Os demais autores declaram não haver conflito de interesses relacionados a este manuscrito. 


\section{REFERÊNCIAS}

1. Suzuki Y, Yeung AC, Ikeno $F$. The representative porcine model for human cardiovascular disease. J Biomed Biotchnol. 2011;2011:195483.

2. Goodman SL. Sheep, pig, and human platelet-material interactions with model cardiovascular biomaterials. J Biomed Mater Res. 1999;45(3):240-50.

3. Shimokawa $\mathrm{H}$, Vanhoutte PM. Impaired endothelium-dependent relaxation to aggregating platelets and related vasoactive substances in porcine coronary arteries in hypercholesterolemia and atherosclerosis. Circ Res. 1989;64(5):900-14.

4. Cromeens DM, Rodgers GP, Minor ST. Warfarin sodium for anticoagulation of atherosclerotic miniature swine. J Invest Surg. 1990;3(2):141-5.

5. Van Belle E, Tio FO, Couffinhal T, Maillard L, Passeri J, Isner JM. Stent endothelialization. Time course, impact of local catheter delivery, feasibility of recombinant protein administration, and response to cytokine expedition. Circulation. 1997;95(2):438-48.

6. Virmani R, Kolodgie FD, Farb A, Lafont A. Drug eluting stents: are human and animal studies comparable? Heart. 2003;89(2):133-8.

7. Farb A, Sangiorgi G, Carter AJ, Walley VM, Edwards WD, Schwartz RS, et al. Pathology of acute and chronic coronary stenting in humans. Circulation. 1999;99(1):44-52.

8. Grewe PH, Deneke T, Machraoui A, Barmeyer J, Muller KM. Acute and chronic tissue response to coronary stent implantation: pathologic findings in human specimen. J Am Coll Cardiol. 2000;35(1):157-63.

9. Schwartz RS, Chronos NA, Virmani R. Preclinical restenosis models and drug-eluting stents: still important, still much to learn. J Am Coll Cardiol. 2004;44(7):1373-85.

10. Schwartz RS, Edelman ER, Carter A, Chronos N, Rogers C, Robinson KA, et al. Drug-eluting stents in preclinical studies: recommended evaluation from a consensus group. Circulation. 2002;106(14):1867-73.

11. Schwartz RS, Edelman E, Virmani R, Carter A, Granada JF, Kaluza GL, et al. Drug-eluting stents in preclinical studies: updated consensus recommendations for preclinical evaluation. Circ Cardiovasc Interv. 2008;1(2):143-53.

12. Kornowski R, Hong MK, Tio FO, Bramwell O, Wu H, Leon $\mathrm{MB}$. In-stent restenosis: contributions of inflammatory responses and arterial injury to neointimal hyperplasia. J Am Coll Cardiol. 1998;31(1):224-30.

13. Suzuki T, Kopia G, Hayashi S, Bailey LR, Llanos G, Wilensky $R$, et al. Stent-based delivery of sirolimus reduces neointimal formation in a porcine coronary model. Circulation. 2001;104(10):1188-93.

14. Gunn J, Arnold N, Chan KH, Shepherd L, Cumberland DC, Crossman DC. Coronary artery stretch versus deep injury in the development of in-stent neointima. Heart. 2002;88(4): 401-5.

15. Takimura CK, Galon MZ, Sojitra P, Doshi M, Aiello V, Gutierrez PS, et al. Estudo da dose excipiente: fármaco com avaliação da hiperplasia neointimal por tomografia de coerência óptica e histopatologia em artérias coronárias porcinas após o emprego do balão eluidor de sirolimus. Rev Bras Cardiol Invasiva. 2012;20(2):133-9.

16. Lemos PA, Laurindo FRM, Morato SP, Takimura C, Campos
CA, Gutierrez PS, et al. Stent coronário de liga cobalto-cromo concebido no Brasil: achados histológicos preliminares em modelo experimental porcino. Rev Bras Cardiol Invasiva. 2007;15(4):378-85.

17. Campos CAHM, Ribeiro EE, Lemos PA, Obregon A, Ribeiro $\mathrm{H}$, Spadaro AG, et al. Resultados clínicos iniciais do primeiro stent de cromo-cobalto concebido no Brasil. Rev Bras Cardiol Invasiva. 2009;17(3):314-9.

18. Takimura CK, Galon MZ, Lopes Junior ACA, Carvalho J, Ferreira SK, Chaves MJF, et al. Avaliação pela tomografia de coerência óptica de stent nacional recoberto com polímero biodegradável eluidor de sirolimus vs. stent eluidor de biolimus A9 em artérias coronárias porcinas. Rev Bras Cardiol Invasiva. 2011;19(2):138-44.

19. Galon MZ, Takimura CK, Carvalho J, Chaves MJF, Lacchini S, Aiello VD, et al. Evolução temporal da proliferação neointimal após implante de dois tipos de stent farmacológico com polímeros biodegradáveis em modelo porcino: avaliação qualitativa por tomografia de coerência óptica sequencial. Rev Bras Cardiol Invasiva. 2012;20(4):413-9.

20. Ribeiro H, Campos C, Lopes A, Esper R, Abizaid A, Meireles $G$, et al. Randomized comparison of the efficacy and safety of a novel DES with biodegradable polymer and cobalt-chromium alloy - INSPIRON I trial [abstract]. Eurolntervention. 2011;7 Suppl M:184.

21. Prado AP, Perez-Martinez C, Cuellas C, Gonzalo-Orden JM, Diego A, Regueiro M, et al. Preclinical evaluation of coronary stents: focus on safety issues. Curr Vasc Pharmacol. 2013;11(1):74-99.

22. Granada JF, Wallace-Bradley D, Win HK, Alviar CL, Builes A, Lev El, et al. In vivo plaque characterization using intravascular ultrasound-virtual histology in a porcine model of complex coronary lesions. Arterioscler Thromb Vasc Biol. 2007;27(2):387-93.

23. Frutkin AD, Mehta SK, McCrary JR, Marso SP. Limitations to the use of virtual histology-intravascular ultrasound to detect vulnerable plaque. Eur Heart J. 2007;28(14):1783-4.

24. Meissner OA, Rieber J, Babaryka G, Oswald M, Reim S, Siebert $U$, et al. Intravascular optical coherence tomography: comparison with histopathology in atherosclerotic peripheral artery specimens. J Vasc Interv Radiol. 2006;17(2 Pt 1):343-9.

25. Mehanna EA, Attizzani GF, Kyono H, Hake M, Bezerra HG Assessment of coronary stent by optical coherence tomography, methodology and definitions. Int J Cardiovasc Imaging. 2011;27(2):259-69.

26. Murata A, Wallace-Bradley D, Tellez A, Alviar C, Aboodi M, Sheehy A, et al. Accuracy of optical coherence tomography in the evaluation of neointimal coverage after stent implantation. JACC Cardiovasc Imaging. 2010;3(1):76-84.

27. Teramoto T, Ikeno F, Otake H, Lyons JK, van Beusekom HM, Fearon WF, et al. Intriguing peri-strut low-intensity area detected by optical coherence tomography after coronary stent deployment. Circ J. 2010;74(6):1257-9.

28. Attizzani GF, Bezerra HG, Chamie D, Fujino $Y$, Spognardi AM, Stanley JR, et al. Serial Evaluation of Vascular Response After Implantation of a New Sirolimus-Eluting Stent With Bioabsorbable Polymer (MISTENT): an optical coherence tomography and histopathological study. J Invasive Cardiol. 2012;24(11):560-8. 